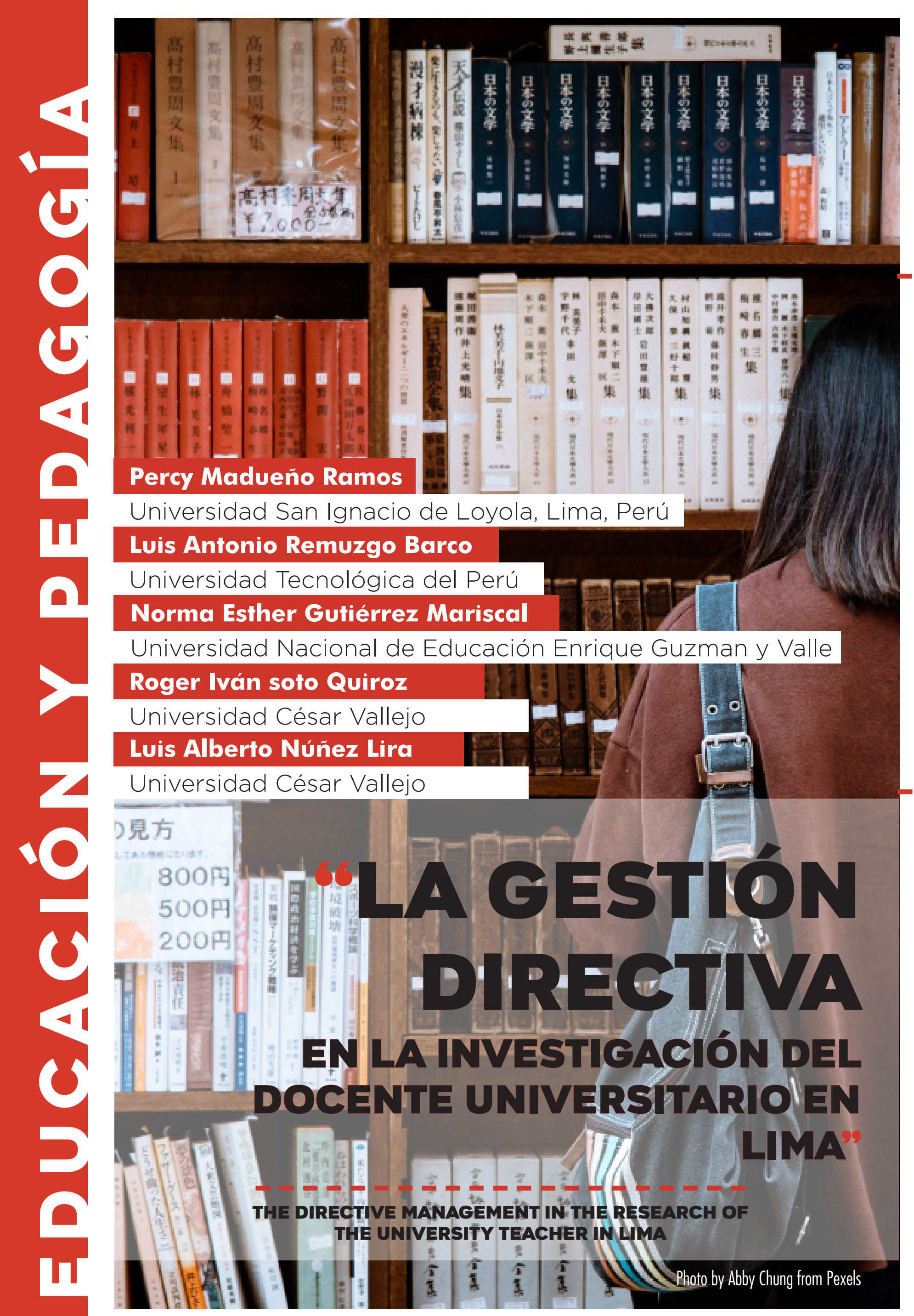


RECIBIDO: $12 / 03 / 2020$

ACEPTADO: $16 / 09 / 2020$

\section{RESUMEN}

El objetivo general del estudio fue determinar la influencia de la gestión directiva en la investigación docente universitaria, en Lima Norte, en el año 2019. La investigación empleó el enfoque cuantitativo, el tipo de investigación fue básica, de nivel explicativo, con la aplicación del diseño no experimental, de corte transversal, para la prueba de hipótesis se utilizó la regresión logística interpretando los resultados del índice del pseudo R cuadrado de Nagelkerke y del nivel de significancia. La población estuvo conformada por 182 docentes universitarios del Programa de Estudios Generales, de una universidad privada societaria, de Lima, Perú, correspondiente al semestre académico 2019-2; y la muestra representativa fue de 124 docentes. Se aplicó la técnica de la encuesta y como instrumentos se utilizaron: la escala de evaluación de la gestión directiva y el cuestionario de investigación docente. Los instrumentos fueron sometidos a la validez de contenido a través del juicio de tres expertos con un resultado de aplicable y el valor de la confiabilidad fue con la prueba Alfa de Cronbach con coeficientes de 0,975 y 0,864 respectivamente, indicándonos una fuerte confiabilidad para ambos instrumentos. Los resultados de la investigación permitieron concluir que: existe influencia de la gestión directiva en la investigación docente universitaria, dado que el nivel de significancia resultó 0,000 y el Pseudo R cuadrado de Nagelkerke fue 0,708 equivalente a un $70,8 \%$ de influencia.

\section{PALABRAS CLAVE}

\section{Gestión directiva, Investigación docente, Formación académica, Motivación, Publicación}

\section{A BSTRACT}

The general objective of the study was to determine the influence of management in university teaching research, in North Lima, in 2019. The research used the quantitative approach, the type of research was basic, at an explanatory level, with the application of the Non-experimental, cross-sectional design, for the hypothesis test, logistic regression was used, interpreting the results of the Nagelkerke pseudo R-square index and the level of significance. The population was made up of 182 university professors from the General Studies Program of a private corporate university in Lima, Peru, corresponding to the 2019-2 academic semester; and the representative sample was 124 teachers. The survey technique was applied and the instruments used were: the evaluation scale of the directive management and the teaching research questionnaire. The instruments were submitted to content validity through the judgment of three experts with an applicable result and the value of reliability was with the Cronbach's alpha test with coefficients of 0.975 and 0.864 respectively, indicating strong reliability for both instruments. The results of the research allowed us to conclude that: there is an influence of the management in university teaching research, given that the level of significance was 0.000 and the Nagelkerke Pseudo R square was 0.708, equivalent to 70.8\% influence.

\section{KEYWORDS}

Management, Teaching research, Academic training, Motivation, Publication. 


\title{
LA GESTIÓN DIRECTIVA EN LA INVESTIGACIÓN DEL DOCENTE UNIVERSITARIO EN LIMA
}

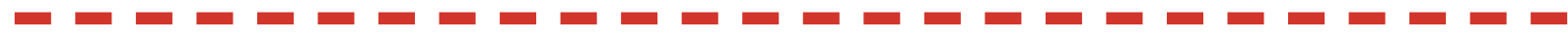 \\ Percy Madueño Ramos \\ Luis Antonio Remuzgo Barco \\ Norma Esther Gutiérrez Mariscal \\ Roger Iván soto Quiroz \\ Luis Alberto Núñez Lira
}

\section{INTRODUCTION}

La investigación parte como realidad problemática considerando que, los tres agentes intervinientes en la educación universitaria: director, docente y estudiante, se interrelacionan mutuamente con la finalidad de formar integralmente a la persona y de brindar una educación de calidad, basada como mínimo en las 8 condiciones básicas de calidad según el requerimiento de licenciamiento de universidades solicitado por la Superintendencia Nacional de Educación Superior Universitaria (Sunedu), considerando que adicionalmente cada universidad de forma voluntaria debería aspirar a la acreditación institucional como es el caso de algunas universidades nacionales e internacionales que ya la tienen. Actualmente, se requieren formas de gestión más eficaces y eficientes para obtener resultados en las universidades, basadas en tres procesos trascendentales: la formación del profesional, la investigación y la extensión universitaria (Veliz, Alonso, Fleitas y Alfonso, 2016).

En el caso de los directores universitarios, una de sus tareas principales es la gestión directiva, basada en cuatro componentes: planificación, organización, dirección y control. Del mismo modo, Rico (2016) considera cuatro fases para la gestión directiva en la educación superior: diseño, planeación, ejecución y evaluación. Asimismo, estas tareas tienen que ver con la gestión de las competencias tanto genéricas como específicas que deben lograr los estudiantes, los contenidos curriculares, los perfiles de ingresante y egresado, la infraestructura pertinente como equipamiento de las aulas, laboratorios, bibliotecas, zonas deportivas, se- lección de docentes, apoyo en el fomento de las investigaciones y producción intelectual de los docentes, exposición de reportes de investigación en eventos nacionales e internacionales, prestación del servicio profesional docente a la comunidad, entre otras. Todas estas tareas mencionadas se logran con un nivel de gestión eficiente de los directores, con una adecuada gestión científica de los procesos pedagógicos para alcanzar una educación universitaria de calidad (Aveiga y Marín, 2013). Por ejemplo, el Ministerio de Educación de Colombia (2015) sustentó que, si se realiza una adecuada gestión en las instituciones educativas teniendo como bases la dirección estratégica y la gestión humana, existe una alta probabilidad que estas instituciones con niveles bajos en calidad logren alcanzar niveles altos de calidad educativa.

Una de las funciones que tienen los docentes universitarios es realizar investigación, siendo ello una debilidad, debido a factores como: falta de tiempo para investigar, poco conocimiento sobre investigación, desinterés por la falta de incentivos y promoción, dificultades para identificar problemas de investigación, falta de presupuesto y publicar, entre otros. Frente a estas dificultades, los directores deben proponer lineamientos claros sobre investigación docente y ayudarlos. La investigación es una problemática en casi todas las universidades peruanas y algunas latinoamericanas debido escaso o nulo financiamiento, no es prioridad universitaria, docentes sin competencias para realizar investigaciones, entre otras (Huayanay, Sánchez, Flores, Bringas y Pérez, 2018; Dáher, Panunzio y Hernández, 2018; Duarte, 
2015; Fernández, 2015). Según Veliz, Alonso, Fleitas y Alfonso (2016), en países de Latinoamérica, "Tanto la educación superior como el desarrollo científico han sido una preocupación central para los Gobiernos de turno" (p.4).

Según Piscoya (2015), el Perú invierte en investigación y desarrollo el 0,15\% del Producto Bruto Interno (PBI), asimismo, a nivel internacional nuestro país alcanza el puesto 117 de un total de 144 instituciones dedicadas a la investigación. No obstante, por otro lado, existe un gran aporte de dinero producto del canon, destinado a las universidades nacionales para la realización de investigación académi$\mathrm{ca}$, pero las universidades o no lo invierten o invierten muy poco de esta suma de dinero, debido a la falta de capacidad y de incentivos para los docentes universitarios. Por ello, las líneas de investigación para pregrado deben estar enmarcadas en resolver problemas cotidianos que consideren los docentes como importantes, del mismo modo las Escuelas Profesionales con asuntos empresariales o de negocios; mientras que en posgrado, existen universidades que figuran en el ranking de universidades que realizan investigación y que tienen pocos estudiantes graduados en posgrado pero con muchos artículos de investigación indexados como la Pontificia Universidad Católica del Perú, Universidad Cayetano Heredia y Universidad Nacional Mayor de San Marcos, mientras que por el contrario, existen otras universidades de tipo societaria que no figuran en el ranking de universidades que realizan investigación y que tienen muchos estudiantes graduados en posgrado pero con ningún artículo de investigación indexado en revistas de impacto.

Del mismo modo, Sunedu (2020) sustenta que, en el ranking de universidades peruanas respecto a la dimensión de investigación está en primer lugar la Universidad César Vallejo con 39313 investigaciones de producción científica; en segundo lugar, la Universidad Católica los Ángeles de Chimbote con 13003 de producción científica, en tercer lugar, la Universidad Nacional de Trujillo con 11811; en cuarto lugar, la Pontificia Universidad Católica del Perú y en quinto lugar : la Universidad Nacional San Agustín de Arequipa con 9323 investigaciones.

Las investigaciones que realiza la universidad debe estar de la mano con la solución de problemas concretos de su comunidad (Delgado, 2016), siendo la prestación de servicios profesionales a la comunidad una más de las funciones de los docentes universitarios, o también llamada aprendizaje servicio, con la finalidad de que la comunidad se desarrolle, ya que la esencia de la existencia de la universidad es extrapolar los conocimientos aprendidos en la universidad en beneficio del desarrollo sostenible de la comunidad. Para ello, los directores establecen estas actividades en el plan operativo y son los docentes en compañía de los estudiantes los que la ejecutan. Entre estas actividades se puede proponer: proyectos de cultura ambiental como mejoramiento de áreas verdes de la comunidad, campañas de reciclaje; proyectos de legislación como ayuda a la comunidad en la creación formal de empresas, denuncias de abusos contra las personas; talleres educativos como sexualidad responsable, habilidades sociales, autoestima, comunicación asertiva; entre otras. En las universidades de Latinoamérica, la gestión universitaria se ha caracterizado por el burocratismo, el centralismo, poco compromiso con el desarrollo de la sociedad y de satisfacer las necesidades sociales de la comunidad (Veliz, Alonso, Fleitas y Alfonso, 2016).

La Gestión Directiva Universitaria, considera que, la gestión tiene que ver con la articulación de: estructura-estrategia-sistemas-estilo-capacidades-gente-objetivos trazados (Bonnefoy, Cerda, Peine, Durán y Ponce, 2019). Mientras que, la gestión directiva universitaria es la forma de gestionar, administrar, gerenciar los recursos humanos, materiales y financieros con la finalidad de lograr los objetivos estratégicos de la universidad, que están relacionados con el desarrollo sostenible de la universidad, el adecuado uso de los recursos humanos (docentes y administrativos), calidad en los servicios y procesos tanto académicos como administrativos, la infraestructura óptima (aulas, laboratorios, auditorios, ambientes deportivos, biblioteca, consultorio psicológico, centro médico, entre otros), cumplimiento de las políticas medioambientales, procesos de licenciamiento, acreditación universitaria, entre otras.

La gestión directiva universitaria cumple una función de soporte y se encarga de que se desarrollen bien las actividades de investigación, docencia y servicios académicos-administrativos. Si la gestión directiva es eficiente, también lo serán la parte académica y administrativa 
(Cárdenas, Farías y Méndez, 2017; Inquilla, Calsina y Velazco, 2017; Proto, 2017).

La gestión directiva universitaria es la forma de gestión para que se alcancen los objetivos estratégicos de calidad y excelencia. La gestión directiva se basa en cuatro procesos: planificación-organización-dirección-control (Cárdenas, Farías y Méndez, 2017).

La gestión directiva son acciones que realizan los directores de una organización con el fin de lograr objetivos administrativos, académicos .Ello involucra planear-evaluar-controlar los procesos de la organización (Miranda, 2016; Bonnefoy, Cerda, Peine, Durán y Ponce, 2019).

Referente a la dimensión gestión directiva universitaria: Gestión institucional, es efectuada por el director está relacionada con la forma de administrar eficiente y eficazmente la universidad, para ello debe planificar las actividades académicas, administrativas, de investigación, de infraestructura, financieras, entre otras. También el director debe ser una persona con competencias cognitivas, manejar un lenguaje apropiado al comunicarse, trabajar en equipo, aplicar estrategias de liderazgo y evaluar permanentemente las actividades que permitirán el logro de los objetivos estratégicos.

Esta dimensión tiene como esencia desarrollar planes de acción con la finalidad de conseguir las metas trazadas por la universidad. Para ello, se necesita realizar diagnósticos, analizar la situación actual, planear estrategias, promover la innovación, el trabajo en equipo, entre otras. Además, de promover docentes líderes y con capacidad de toma de decisiones (Cárdenas, Farías y Méndez, 2017).

En esta dimensión también se toma en cuenta el liderazgo en la dirección de la universidad relacionado con la gestión del modelo educativo adoptado, la formación continua de los docentes en cuanto a capacitaciones y actualizaciones, actividades orientadas al conocimiento, la investigación y la docencia, con la finalidad de lograr la satisfacción de las necesidades de los estudiantes, docentes y comunidad universitaria (Cárdenas, Farías y Méndez, 2017).

Asimismo, es importante el proceso de evaluación ejecutada en la universidad tanto en lo académico, administrativo, entre otros, con la finalidad de lograr la excelencia y calidad educativa, es por ello que la universidad se somete a procesos de evaluación institucional como el licenciamiento y la acreditación (Cárdenas, Farías y Méndez, 2017). Al respecto, Bonnefoy, Cerda, Peine, Durán y Ponce (2019) consideran que, el control en la gestión directiva es una de las tareas de los directores universitarios que consiste en evaluar la investigación, gestión y docencia.

Referente a la dimensión gestión directiva universitaria: Valórica actitudinal, esta dimensión se refiere a que el director debe poseer y dar muestras de valores morales como la puntualidad, responsabilidad, orden, perseverancia, solidaridad, respeto, honestidad, entre otros. Además de ser una persona que aplica la éti$\mathrm{ca}$, ser una buena persona, de tal manera que eso contagie a los demás integrantes de la universidad.

Una de las teorías relacionadas con la gestión directiva es la calidad total, un sistema eficaz capaz de integrar el desarrollo, mantenimiento y mejoramiento de todos los trabajadores de la universidad deben realizar con la finalidad de brindar productos y servicios académicos y administrativos que satisfagan lo que esperan los usuarios (Bonnefoy, Cerda, Peine, Durán y Ponce, 2019).

La teoría de las competencias: cognitivas, procedimentales y actitudinales contribuyen con la gestión directiva, puesto que las competencias de cada trabajador y de los equipos de trabajo de la universidad hacen que los aspectos académicos y administrativos sean de calidad. Entre las competencias que desarrollan las personas se tiene: competencias de comunicación, de pensamiento crítico, de ética, de valores, de relaciones interpersonales, de gestión, de liderazgo, entre otras (Bonnefoy, Cerda, Peine, Durán y Ponce, 2019; Muñoz y Araya, 2017).

Otra teoría que sustenta la gestión directiva es la teoría de los modelos de gestión de calidad como el sistema ISO (International Organization for standardization), donde todo aquello que está escrito debe verificarse en cada actividad de un proceso determinado; modelo de gestión de calidad EFQM (European Foundation for Quality Management), donde se busca satisfacer a todos los clientes y la comunidad universitaria, además trae como efectos positivos que los costos se reduzcan, satisfacción óptima, producción adecuada y nivel de ren- 
tabilidad muy buena (Lizarzaburu, 2016; Barrios, Ricard y Fernández, 2016).

También la teoría de sistemas sociales, sustenta que la organización es un conjunto complejo pero sistemático, donde todas las partes que la conforman interactúan entre ellas y se adaptan a los cambios con la finalidad de lograr los objetivos (Segredo, 2016).

La investigación docente universitaria se define como la actividad de descubrir nuevos conocimientos por medio de la investigación realizada por los docentes universitarios, contribuyendo de este modo al desarrollo de la sociedad. Estas investigaciones pueden ser de enfoque cuantitativo, cualitativo o mixto; de nivel exploratorio, descriptivo, correlacional o explicativo; de diseños experimentales como no experimentales. El objetivo es que los docentes investiguen principalmente en su área de formación, para ello deben mostrarse con actitudes positivas hacia la investigación, con dominio temático y metodológico, con motivación permanente, con visión proactiva, con gusto por la investigación, involucrarse en la investigación (Manfra, 2019).

Para Rodríguez (2017) la investigación es una actividad inherente, irrenunciable, esencial, natural, que toda universidad tiene que realizar por considerarse una institución de educación superior. Existen algunas universidades donde se prioriza la docencia antes que la investigación y otras donde la investigación es la tarea principal por lo que todos los docentes realizan investigación. Las contribuciones de las investigaciones no solo deben ser para la comunidad científica sino también para el desarrollo de la sociedad al resolver problemáticas relacionadas con la industria, educación, salud, entre otras.

Arellano, Hermoza, Elías y Ramírez (2017) manifiestan que, el término investigación se refiere a la realización de la actividad intelectual y experimental de forma sistemática con la finalidad de incrementar los conocimientos referentes a una determinada materia.

La universidad propone líneas de investigación para cada carrera profesional en sus currículos, con la finalidad de contribuir a la solución de problemas que se presentan en las regiones del país. Los estudiantes y docentes son los encargados de desarrollar los proyectos e informes de investigación de tipo básica, aplicada o tecnológica. Por otro lado para ser considerado como docente investigador Universitario, el docente debe generar conocimiento e innovación. Es seleccionado después de aprobar la convocatoria para ser docente investigador y ser reconocido mediante resolución rectoral y resolución del vicerrectorado de investigación de la universidad.

Las investigaciones en la universidad son de dos tipos: la investigación formativa y la investigación

Referente a la dimensión investigación del doC universitario: Formación académica en investigación. Esto se define como el nivel académico que tiene el docente universitario sobre la investigación científica, es decir qué tan preparado se encuentra el docente para realizar una investigación. Esta preparación tiene que ver con los conocimientos que posee sobre enfoques de investigación, tipos de investigación, metodología, estadística aplicada a la investigación, manejo de fuentes de información, manuales de redacción, conocimiento de las líneas de investigación y actualización en investigación.

Arellano, Hermoza, Elías y Ramírez (2017) sostienen que, la formación en investigación son todas las actividades encaminadas al desarrollo de conocimientos, habilidades y actitudes necesarias para que tanto los docentes como los estudiantes desempeñen favorablemente la investigación científica o bien en el área académica o bien en el área productiva. Asimismo, Jiménez (2018) argumenta que, los docentes universitarios que se dedican a la investigación tienen responsabilidades como: la creación, desarrollo y socialización del conocimiento; además tienen la tarea de crear equipos de investigación con estudiantes y docentes nacionales e internacionales, de esta manera se fomenta las redes de investigación y la iniciación científica como parte de su formación académica en la investigación científica.

Muñoz y Garay (2015) sustentan que, el perfil del docente universitario res : ser comprometidos con la educación, con competencias profesionales adecuadas tanto en conocimientos-habilidades-actitudes para la enseñanza como para la investigación, con autonomía para tomar decisiones, con disposición a cambiar, con ética, con valores, capaz de trabajar en equipo, líder en el aula y fuera de ella, en- 
tre otras. Asimismo, Gonzáles (2014) sostiene la importancia de la formación académica en la redacción de escritos científicos que traerá como consecuencia el incremento de las publicaciones de dichas investigaciones.

Referente a la dimensión investigación docente universitario: Motivación por la investigación. Esta dimensión se define como el interés que tiene el docente por realizar una investigación científica (Li-fang, Mingchen, Dorothy \& Yunfeng, 2019; Masoud \& Hiwa, 2018). Este interés tiene que ver con las motivaciones que toma en cuenta el docente al momento de elaborar su investigación, estas motivaciones pueden ser tres: (1) Motivaciones intrínsecas, como el deseo de desarrollo profesional, el anhelo por descubrir nuevos conocimientos. (2) Motivaciones extrínsecas, como obtener incentivos económicos, deseo de publicar en revista indizada. (3) Motivaciones trascendentes, como la aspiración por contribuir con el desarrollo de la sociedad. Referente a la dimensión 3 de investigación docente universitaria: Realización y publicación de trabajos de investigación. Esto se define como la elaboración de productos de investigación científica que realizan los docentes universitarios sobre temas que le interesan, inicia con la elaboración de un proyecto de investigación, continúa con el trabajo de campo, luego la elaboración del artículo de investigación y su destino final es la publicación del artículo en revistas indexadas de nivel básico como Latindex, intermedio como Scielo y de impacto como Scopus o Web of Science.

Según la Sunedu (2018), la forma de evaluar la investigación universitaria en nuestro país es mediante el cumplimiento de estándares internacionales vinculados con la publicación de investigaciones cuyos indicadores son: (1) Número de documentos citables, por ejemplo, en el 2015 en América Latina y El Caribe, Brasil tuvo 57033 publicaciones, México 16930, Argentina 10746, Chile 9540, Colombia 6990, Cuba 1631, Perú 1610, Venezuela 1345, Ecuador 1328 y Uruguay 1115; (2) Número de revistas académicas indexadas por Scopus, por ejemplo, nuestro país aumento de 4 a 7 del año 2010 al 2015, mientras que Brasil aumento de 262 a 332 en el mismo periodo.

Sunedu (2018), exige a las universidades, como parte de los 8 indicadores de calidad educativa, que los docentes tengan como mínimo el grado de magíster y además que tengan publicaciones de investigaciones en re- vistas indexadas. El que un docente realice investigaciones implica que posee conocimientos sobre investigación, que tiene experiencia realizando investigación, pero la realidad es que en algunas universidades, los docentes tienen tanta carga académica como docente a tiempo completo, con 30 horas de enseñanza y 10 horas para realizar trabajos administrativos, que en vez de emplearlos para investigar, lo utilizan para otras tareas netamente administrativas que lo alejan de sus funciones investigativas.

\section{METODOLOGÍA}

El enfoque empleado en la investigación es el cuantitativo. Se usa la medición numérica para encontrar resultados y el análisis estadístico para describir los resultados y para probar las hipótesis (Valderrama, 2013; Hernández, Fernández y Baptista, 2010).

Es una investigación básica, o investigación fundamental, pura o teórica, su esencia es descubrir nuevos conocimientos científicos por medio del recojo de información de la realidad (Valderrama, 2013; Tamayo, 2010), en la presente investigación se recoge información desde la percepción de los docentes universitarios de la universidad en estudio.

El presente estudio emplea el diseño no experimental, correlacional-causal, de corte transversal (Valderrama, 2013). Es no experimental ya que no se realizan experimentos, ni manipulan variables, no hay grupo control ni experimental (Soto, 2015; Carrasco, 2010). El estudio es de corte transversal en vista que la recolección de los datos es en un solo momento. El método usado fue el hipotético-deductivo.El que consiste en plantear hipótesis en la investigación como una forma de afirmar respuestas tentativas, para luego demostrar tales hipótesis (aceptar o rechazar las hipótesis) a traves de la deducción de conclusiones producto de los resultados inferenciales obtenidos de las pruebas estadísticas aplicadas (Valderrama, 2013; Soto, 2015 ).

La población estuvo conformada por 182 docentes y la muestra fue probabilística y estuvo conformada por 124 docentes.Para la selección de los participantes del estudio se aplicó la técnica de muestreo aleatorio simple. 


\section{MÉTODOS DE ANÁLISIS DE DATOS}

En el caso de los resultados del nivel de gestión directiva, el nivel de investigación docente y los niveles de las dimensiones en tablas de distribución de frecuencias para variables cualitativas, además de los gráficos de barras simples presentados como figuras.

Para el caso de la estadística inferencial, ya que ambas variables son de naturaleza cualitativa y de escala ordinal, se utilizó estadísticos no paramétricos, en este caso la Regresión Logística para contrastar las hipótesis.

\section{RESULTADOS}

Luego de procesar la información y de aplicar el test de la gestión directiva nos indican que el $24,2 \%$ presenta nivel deficiente, $11,3 \%$ presenta un nivel moderado y el 64,5\% presenta nivel eficiente; en la dimensión gestión institucional nos indican que el 81,5\% presenta nivel deficiente, $1,6 \%$ presenta un nivel moderado y el $16,9 \%$ presenta nivel eficiente; en la dimensión valórica actitudinal nos indican que el 91,9\% presenta nivel deficiente, 0,8\% presenta un nivel moderado y el 7,3\% presenta nivel eficiente.

Tabla 1

Niveles de la gestión directiva y dimensiones

\begin{tabular}{rcccccc} 
& \multicolumn{2}{c}{$\begin{array}{c}\text { Gestión Directiva } \\
\text { Universitaria }\end{array}$} & \multicolumn{2}{c}{ Gestión institucional } & \multicolumn{2}{c}{ Valórica actitudinal } \\
& $\mathbf{f}$ & $\mathbf{\%}$ & $\mathbf{f}$ & $\mathbf{\%}$ & $\mathbf{f}$ & $\%$ \\
\hline Deficiente & 30 & 24,2 & 101 & 81,5 & 114 & 91,9 \\
\hline Moderada & 14 & 11,3 & 2 & 1,6 & 1 &, 8 \\
\hline Eficiente & 80 & 64,5 & 21 & $16,9 \mathrm{~W}$ & 9 & 7,3 \\
\hline Total & 124 & 100,0 & 124 & 100,0 & 124 & 100,0 \\
\hline
\end{tabular}

Figura 1

Niveles de la gestión directiva y dimensiones

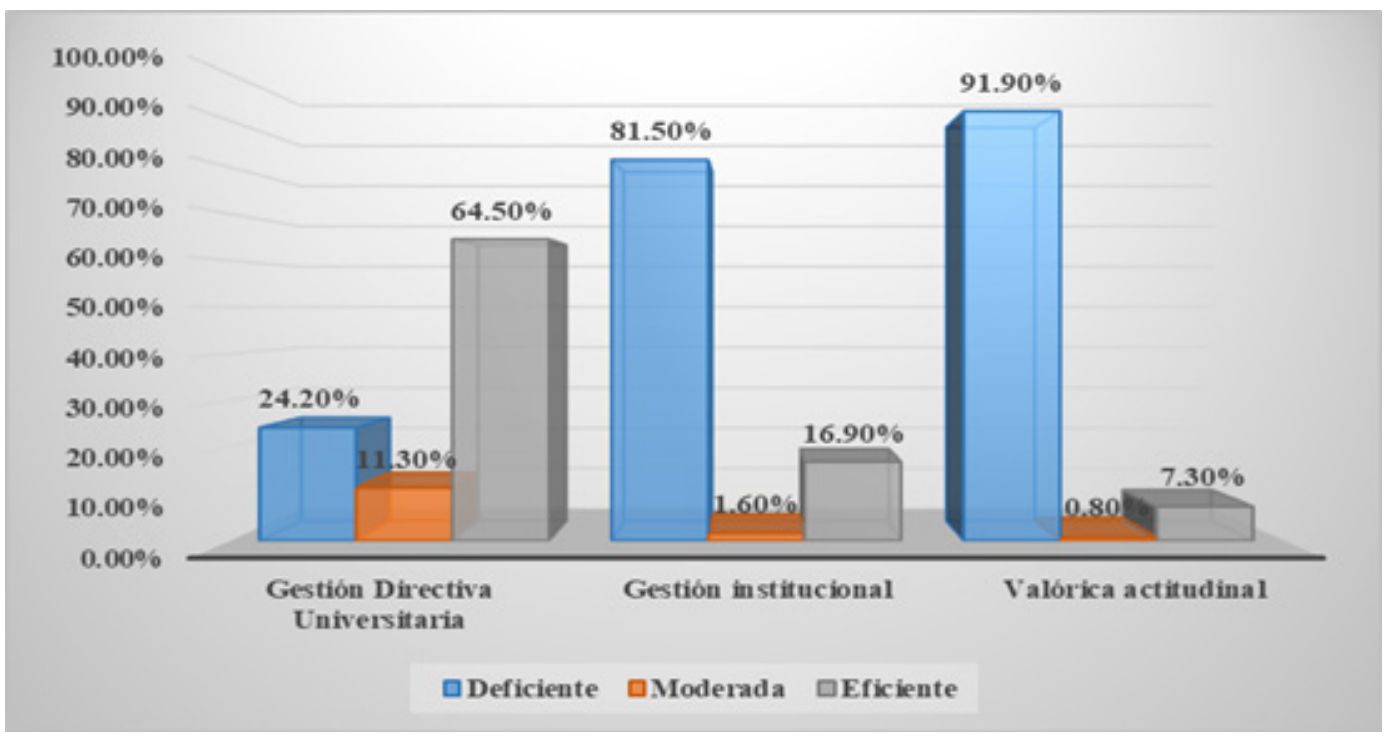


De la figura anterior el 62,1\% presenta nivel deficiente, $37,9 \%$ presenta un nivel moderado; en la dimensión formación académica en investigación nos indican que el $86,3 \%$ presenta nivel deficiente, $13,7 \%$ presenta un nivel moderado; en la dimensión motivación por la investigación en investigación nos indican que el $27,4 \%$ presenta nivel deficiente, $60,5 \%$ presenta un nivel moderado, 12,1\% presenta un nivel eficiente; en la dimensión realización y publicación de trabajos de investigación en investigación nos indican que el 75,0\% presenta nivel deficiente, $25,0 \%$ presenta un nivel moderado.

Tabla 2

Niveles de la investigación docente y dimensiones

\begin{tabular}{rcccccccc} 
& $\begin{array}{c}\text { Investigación } \\
\text { docente } \\
\text { universitaria }\end{array}$ & $\begin{array}{c}\text { Formación } \\
\text { académica en } \\
\text { investigación }\end{array}$ & $\begin{array}{c}\text { Motivación por la } \\
\text { investigación }\end{array}$ & $\begin{array}{c}\text { Realización y } \\
\text { publicación de } \\
\text { trabajos de } \\
\text { investigación }\end{array}$ \\
\hline Deficiente & 77 & 62,1 & 107 & 86,3 & 34 & 27,4 & 93 & 75,0 \\
\hline Moderada & 47 & 37,9 & 17 & 13,7 & 75 & 60,5 & 31 & 25,0 \\
\hline Eficiente & 0 & 0,0 & 0 & 0,0 & 15 & 12,1 & 0 & 0,0 \\
\hline Total & 124 & 100,0 & 124 & 100,0 & 124 & 100,0 & 124 & 100,0 \\
\hline
\end{tabular}

Figura 2

Niveles de la investigación docente y dimensiones

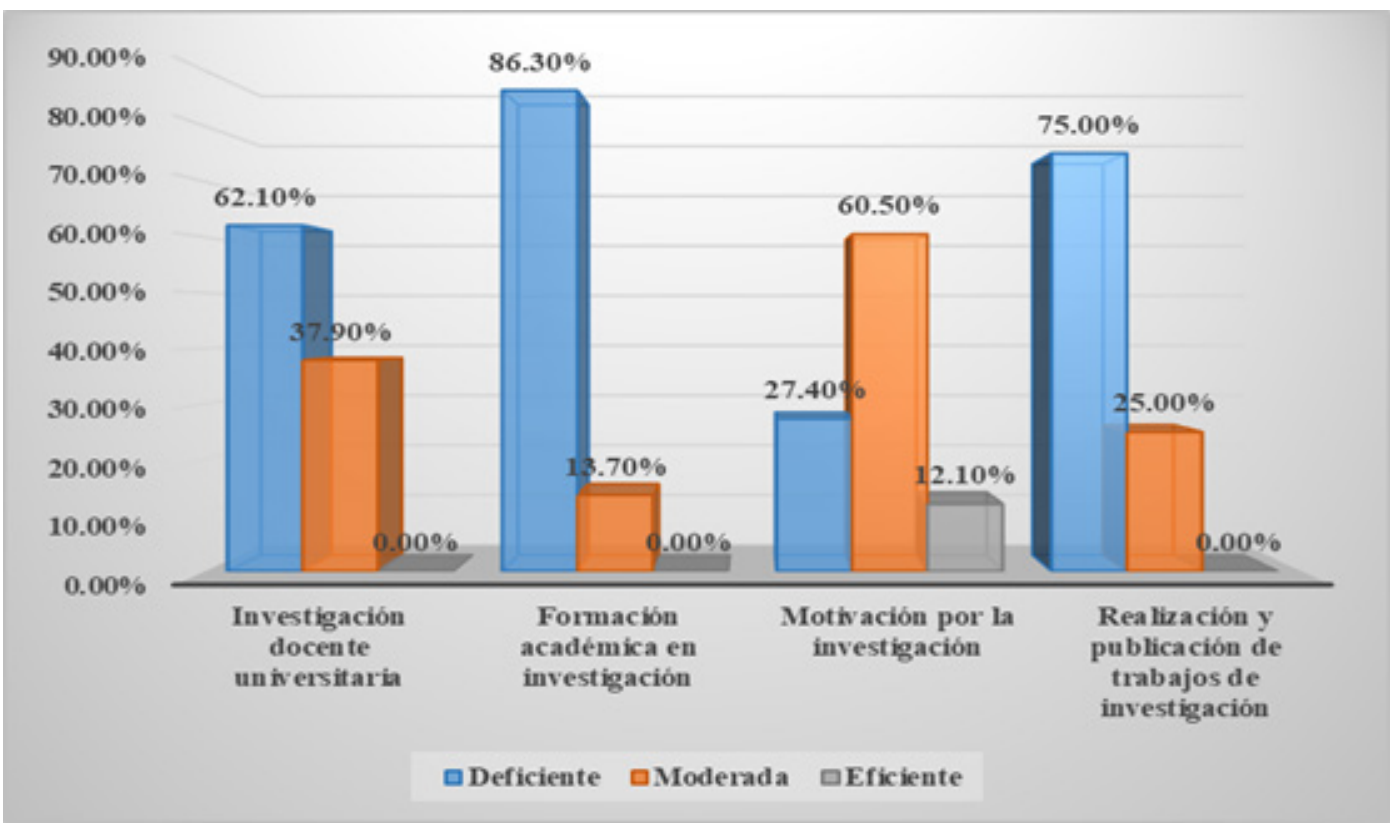


La influencia de la gestión directiva universitaria en la investigación del docente universitario, está siendo explicada por la variable independiente de acuerdo al índice del pseudo cuadrado de Nagelkerke de 0,708, es decir, $70,8 \%$ de influencia; la hipótesis específica 1, la influencia de la gestión directiva universitaria en la formación académica en investigación, nos indica que la variable dependiente está siendo explicada por la variable independiente de acuerdo al índice del pseudo cuadrado de Nagelkerke de 0,671, es decir, 67,1\% de influencia; la hipótesis específica 2, la influencia de la gestión directiva universitaria en la mo- tivación por la investigación, nos indica que la variable dependiente está siendo explicada por la variable independiente de acuerdo al índice del pseudo cuadrado de Nagelkerke de 0,655, es decir, 65,5\% de influencia; la hipótesis específica 3, la influencia de la gestión directiva universitaria en la realización y publicación de trabajos de investigación, nos indica que la variable dependiente está siendo explicada por la variable independiente de acuerdo al índice del pseudo cuadrado de Nagelkerke de 0,617, es decir, 61,7\% de influencia.

Tabla 3

Niveles de la investigación docente y dimensiones

\begin{tabular}{|c|c|c|c|c|c|c|}
\hline Hipółesis & Variables & $\begin{array}{l}\text { Logaritmo de la } \\
\text { verosimilitud }-2\end{array}$ & Chicuadrado & Sig. & $\begin{array}{c}\text { Pseudo R } \\
\text { cuadrado } \\
\text { Nagelkerke }\end{array}$ & $\begin{array}{l}\text { Influencia } \\
\text { x } 100 \%\end{array}$ \\
\hline $\begin{array}{l}\text { Hipótesis } \\
\text { general }\end{array}$ & $\begin{array}{l}\text { Gestión Directiva } \\
\text { Universitaria* } \\
\text { Investigación } \\
\text { docente universitaria }\end{array}$ & 27,581 & 91,051 & 0,000 & 0,708 & $70,8 \%$ \\
\hline $\begin{array}{l}\text { Hipótesis } \\
\text { específica } \\
1\end{array}$ & $\begin{array}{l}\text { Gestión Directiva } \\
\text { Universitaria* } \\
\text { Formación académica } \\
\text { en investigación }\end{array}$ & 16,543 & 57,195 &, 047 & 0,671 & $67,1 \%$ \\
\hline $\begin{array}{l}\text { Hipótesis } \\
\text { específica } \\
2\end{array}$ & $\begin{array}{l}\text { Gestión Directiva } \\
\text { Universitaria* } \\
\text { Motivación por la } \\
\text { investigación }\end{array}$ & 92,332 & 98,888 & 0,000 & 0,655 & $65,5 \%$ \\
\hline $\begin{array}{l}\text { Hipótesis } \\
\text { específica } \\
3\end{array}$ & $\begin{array}{l}\text { Gestión Directiva } \\
\text { Universitaria* } \\
\text { Realización y publi- } \\
\text { cación de trabajos de } \\
\text { investigación }\end{array}$ & 28,360 & 66,758 & 0,012 & 0,617 & $61,7 \%$ \\
\hline
\end{tabular}

\section{DISCUSIÓN}

Referente al objetivo general que fue determinar la influencia de la gestión directiva en la investigación docente universitaria, se pudo demostrar que la variable investigación docente fue explicada por la variable gestión directiva de acuerdo al índice del pseudo cuadrado de Nagelkerke cuyo valor fue 0,708, lo que indicó un $70,8 \%$ de influencia. Siendo este un resultado bastante elevado, deduciéndose que la gestión del director es un factor muy importante para que los docentes realicen eficientemente la tarea de la investigación, ya sea de forma voluntaria o como parte de sus horas académicas programadas. Es por ello que,
Aveiga y Marín (2013) consideran que el apoyo en el fomento a la investigación de los docentes se logra con la gestión eficiente de los directores. Asimismo, Rico (2016) y Campos (2015) sustentan que existe una relación significativa entre la gestión y la investigación docente; además, la universidad es la encargada de gestionar a través de sus autoridades que los docentes realicen investigaciones y participen como ponentes de reportes de investigación en eventos académicos como congresos, no obstante, estos reportes deben ser publicados en libros o revistas de investigación. 
Los resultados descriptivos de la variable gestión directiva universitaria muestran al $64 \%$ de docentes quienes perciben una gestión eficiente, el $11,3 \%$ la considera como moderada y el 24,2\% observan una gestión deficiente. Así la gestión directiva tiene una tendencia al nivel eficiente, por tanto existe una tendencia a la calidad educativa y por ende en investigación docente, tal como lo sustenta el Ministerio de Educación de Colombia (2015) al fundamentar que cuando existe una adecuada gestión en las instituciones educativas que tienen como pilares a la dirección estratégica y la gestión humana, es muy probable que estas instituciones con niveles bajos en calidad logren alcanzar niveles altos de calidad educativa. La gestión directiva universitaria tiene como funciones desarrollar bien las actividades de investigación, docencia y servicios académicos-administrativos, que son las tareas principales de la universidad; si la gestión directiva es eficiente, entonces los aspectos académicos y administrativos también serán eficientes (Cárdenas, Farías y Méndez, 2017; Inquilla, Calsina y Velazco, 2017; Proto, 2017). Los resultados señalan que existe una gestión eficiente, por lo tanto, los objetivos planteados en la universidad se cumplirán, así como lo sostiene Miranda (2016). Un aspecto importante en la gestión directiva es el logro de los objetivos trazados (Bonnefoy, Cerda, Peine, Durán y Ponce, 2019). Asimismo, Cárdenas, Farías y Méndez (2017), en su investigación demostraron que la gestión realizada por el director impacta positivamente en el desarrollo de proyectos de innovación educativa, cumpliendo de este modo un objetivo planteado por la universidad. También Plasencia (2017) en su estudio encontró que la gestión directiva tenía un nivel bajo correspondiente al $8.5 \%, 73,5 \%$ en nivel moderado y el $18 \%$ indicó un nivel alto; se aprecia una tendencia al nivel moderado, mientras que si lo comparamos con los resultados de la presente investigación difieren puesto que el nivel de gestión directiva es eficiente.

Los resultados descriptivos de la variable investigación docente indican que el $62,1 \%$ de docentes presenta un nivel deficiente, mientras que el $37,9 \%$ manifiesta un nivel moderado. El detalle que resalta en estos resultados es que ninguno de los docentes percibe como nivel eficiente la realización de investigaciones, se aprecia que los docentes no realizan investigaciones, incumpliendo una de las tareas esenciales de la universidad. Tal como lo sostienen Huayanay, Sánchez, Flores, Bringas y Pérez (2018); Dáher, Panunzio y Hernández (2018); Duarte (2015); Piscoya (2015) y Fernández (2015) al considerar la investigación como una problemática latinoamericana, por diversos motivos como poca inversión económica, no es una actividad prioritaria, falta de formación en investigación por parte de los docentes, entre otros. Rodríguez (2017) sustenta que, la investigación es una actividad esencial de la universidad, por lo tanto, debe tener tanta importancia como la docencia y la responsabilidad social universitaria. Muñoz y Garay (2015); Gonzáles (2014) sostienen que como parte del perfil del docente universitario es contar con competencias para la investigación científica y posteriormente realizar las publicaciones pertinentes en revistas de investigación. Del mismo modo, Coronado (2018) analiza la realidad universitaria peruana en cuanto a investigación docente y sustenta que la Sunedu exige que las universidades logren el licenciamiento, donde una de las exigencias es la producción científica de sus docentes. Al presentar un nivel deficiente en investigación docente, entonces no se resuelven problemas concretos que ocurren en la sociedad; al respecto Delgado (2016) sustenta que las investigaciones que efectúa la universidad tiene el propósito de solucionar problemas de su realidad; igualmente Martí, Calderón y Fernández (2018) fundamentan que las investigaciones que realizan los docentes deben buscar solucionar problemas reales de la comunidad de tal manera que se mejore la calidad de vida de las personas y que se desarrolle integralmente la sociedad. Existe coincidencia con los resultados encontrados por Rivera, Espinoza y Valdés (2017), dado que la tarea que priorizaba dicha universidad en estudio fue la docencia y no la tarea de investigación científica por parte de los docentes. Burns y Westmacott (2018); Wyalt y Dikilitas (2015); Yuan y Lee (2014) descubrieron algunas alternativas para que los docentes sean investigadores, entre ellas son: Capacitarse a través de talleres de investigación científica de manera interna o externa a la universidad, inscribirse en programas de posgrado que priorice la práctica de la investigación.

Referente al objetivo específico 1 se pudo demostrar que la variable formación académica en investigación fue explicada por la variable gestión directiva de acuerdo al índice del pseudo cuadrado de Nagelkerke cuyo valor fue 0,671 , lo que indicó un $67,1 \%$ de influen- 
cia. Siendo este un porcentaje elevado, concluyendo que la gestión directiva es un factor trascendente en la formación académica en investigación por parte de los docentes. Estos resultados coinciden con las investigaciones de Rico (2016) y Campos (2015) quienes demostraron que existe un vínculo significativo entre la gestión y la formación docente en sus cuatro dimensiones: calidad científica, desarrollo pedagógico, investigación docente y prestación del servicio docente a la comunidad.

Según los resultados descriptivos sobre la formación académica en investigación, el 86,3\% la percibe como nivel deficiente, el 13,7\% en el nivel moderada, mientras que ninguno alcanza el nivel eficiente. Esta deficiente formación en investigación coincide con lo planteado por Piscoya (2015), al afirmar que los docentes no investigan porque les falta la formación y capacidad para investigar. Arellano, Hermoza, Elías y Ramírez (2017) fundamentan que, la formación en investigación contribuye a que los docentes se desempeñen adecuadamente en la producción de investigaciones científicas. Del mismo modo, coinciden Muñoz y Garay (2015) al sostener que, el perfil del docente universitario debe contener competencias profesionales adecuadas tanto en conocimientos-habilidades-actitudes para la enseñanza como para la investigación. También, Gonzáles (2014) sostiene que como parte de la formación académica de los docentes son las destrezas en la redacción de escritos científicos, por consiguiente, facilitará en la publicación de sus investigaciones.

Referente al objetivo específico 2 se pudo demostrar que la variable motivación por la investigación fue explicada por la variable gestión directiva de acuerdo al índice del pseudo cuadrado de Nagelkerke cuyo valor fue 0,655, lo que indicó un $65,5 \%$ de influencia. Siendo este un porcentaje elevado, concluyendo que la gestión directiva es un factor muy trascendente en la motivación por la investigación.

Según los resultados descriptivos referente a la motivación por la investigación, el 27,4\% de docentes presenta un nivel deficiente, el $60,5 \%$ un nivel moderado y el $12,1 \%$ en el nivel eficiente. Se aprecia que la mayoría de docentes tienen poca o ninguna motivación para realizar investigaciones científicas, coincidiendo con lo argumentado por Piscoya (2015) quien manifiesta que los docentes universitarios no investigan por la falta de incentivos para inves- tigar. También, Camacho, Escalante, Quispe y Salazar (2014) en su investigación descubrieron que las motivaciones extrínsecas tienen que ver principalmente con las condiciones físicas y ambientales en el trabajo, los bajos sueldos y el exceso de carga académica semanal, lo que muchas veces dificulta la actividad investigativa. Con una carga académica elevada para un docente a tiempo completo, que algunas veces es de 30 a 35 horas semanales en las aulas, se hace difícil que los docentes realicen investigaciones científicas por no contar con el tiempo necesario, solo algunos la realizarán, pero por automotivación más no porque la universidad tiene como política el fomento de la investigación en sus docentes.

Referente al objetivo específico 3, se pudo demostrar que la variable realización y publicación de trabajos de investigación fue explicada por la variable gestión directiva de acuerdo al índice del pseudo cuadrado de Nagelkerke cuyo valor fue 0,617 , lo que indicó un $61,7 \%$ de influencia. Concluyendo que la gestión directiva es un factor muy trascendente en la realización y publicación de trabajos de investigación de los docentes.

Según los resultados descriptivos referentes a la realización y publicación de trabajos de investigación por parte de los docentes, $75 \%$ de ellos considera que es deficiente, el 25\% indica que es moderado y ninguno señala que es eficiente. En muchas universidades peruanas la mayoría de docentes no realizan publicaciones, no obstante, según la Sunedu (2018) existen algunas universidades como la Pontificia Universidad Católica del Perú con 100\% de producción científica de sus docentes, además de la Universidad Cayetano Heredia con un 69\%, siguiendo la Universidad Nacional Mayor de San Marcos con un 55\%. Gonzáles (2014) también considera importante que los docentes realicen las publicaciones de sus investigaciones, además de difundirlas en eventos tanto nacionales como internacionales, de este modo el docente puede formar redes entre otras universidades o instituciones académicas para desarrollar investigación científica.

\section{REFERENCIAS}

Arellano-Sacramento, C., Hermoza-Moquillaza, R. V., Elías-Podestá, M., \& Ramírez-Jul$\mathrm{ca}, \mathrm{M}$. (2017). Actitud hacia la investigación de estudiantes universitarios en Lima, Perú. FEM: Revista de la Fundación Educa- 
ción Médica, 20(4), 191-197. Recuperado en 16 de octubre de 2018, de http:// scielo.isciii.es/scielo.php? script $=$ sci arttext\&pid =S2014-98322017000400006\&ln$\mathrm{g}=$ es\&tlng $=$ es.

Aveiga, V. I., \& Marín, C. (2013). La gestión de la superación del directivo universitario. Necesidad social de un modelo. Humanidades Médicas, 13(3), 639-654. Recuperado en 06 de junio de 2019, de http:// scielo.sld.cu/scielo.php? script $=$ sci arttext\&pid =S1 727-81202013000300005\&ln$\mathrm{g}=$ es\&tlng $=\mathrm{es}$.

Barrios, No., Ricard, M., \& Fernández, R. (2016). La definición de funciones en la gestión de la calidad de los procesos universitarios. Cofin Habana, 10(2), 95-114. Recuperado en 27 de noviembre del 2019, de http:// scielo.sld.cu/scielo.php? script $=$ sci arttext\&pid =S2073-60612016000200005\&ln$\mathrm{g}=\mathrm{es} \& \mathrm{t} \operatorname{lng}=\mathrm{es}$.

Bonnefoy, C., \& Cerda, G., \& Peine, S., \& Durán, M., \& Ponce, Y. (2019). Gestión Directiva Universitaria: Un Instrumento para su Evaluación. Revista de Psicología, XIII (2), 63-82.

Burns, A., \& Westmacott, A. (2018). Teacher to Researcher: Reflections on a New Action Research Program for University EFL Teachers. Profile Issues in Teachers' Professional Development, 20(1), 15-23. DOl: https://dx. doi.org/10.15446/profile.v20n 1.66236

Camacho, V., Escalante, V., Quispe, L. y Salazar, M. (2014). Motivación laboral hacia la investigación científica del profesional de enfermería asistencial en un hospital nacional. Revista enfermería Herediana, 7(2), 111-117. Recuperado de http://www.upch. edu.pe/vrinve/dugic/revistas/index.php/ RENH/article/viewFile/2560/2465

Campos, N. (2015). ¿'Por qué es importante que el personal docente investigue? Reflexión a partir de datos de una experiencia de investigación etnográfica. Revista actualidades investigativas en educación, 15(3), 1-16. DOI: http://dx.doi.org/10.15517/ aie.v15i3.21071

Cárdenas, C., \& Farías, G., \& Méndez, G. (2017). ¿̇Existe Relación entre la Gestión Administrativa y la Innovación Educativa?
Un Estudio de Caso en Educación Superior. REICE. Revista Iberoamericana sobre Calidad, Eficacia y Cambio en Educación, 15 (1), 19-35.

Carrasco, D. (2010). Metodología de la investigación científica. Lima, Perú: San Marcos.

Castro, M. y Alves, D. (2017). Teaching, research and extension at the Universidade Federal de Viçosa: origin and institutional trajectory (1926-1988). Revista Brasileira de Educação, 22(70), 752-773. DOI: https://dx.doi.org/10.1590/s141324782017227038

Coronado, D. (2018). EL rol de las universidades peruanas frente a la investigación y el desarrollo tecnológico. Propósitos y Representaciones, 6(2), 703-737. DOl: http:// dx.doi.org/10.20511/pyr2018.v6n2.244

Dáher, J. E., Panunzio, A. P., \& Hernández, M. I. (2018). La investigación científica: una función universitaria a considerar en el contexto ecuatoriano. EDUMECENTRO, 10(4), 166-179. Recuperado en 20 de junio de 2019, de http://scielo. sld.cu/scielo. php? script = sci arttext\&pi$d=$ S2077-2874201800040001 1\&ln$\mathrm{g}=\mathrm{es} \&$ tlng $=\mathrm{es}$.

Delgado, L. (2016). Universidad e Investigación. Revista Estomatológica Herediana, 26(2), 61 -62. https://dx.doi.org/10.20453/ reh.v26i2.2866

Duarte, M. (2015). Importancia de la investigación científica en la vida universitaria. Revista Científica de la UCSA, 2(2) 3-5. Doi: 10.18004/ucsa/24098752/2015.002(02)003-005

Fernández, C. (2015). La investigación científica en Uruguay. Revista Médica del Uruguay, 37 (4), 229-230. Recuperado en 20 de junio de 2019, de http://www.scielo. edu.uy/scielo. php? script=sci arttext\&pi$d=S 1688-03902015000400001 \& \mid n-$ $\mathrm{g}=\mathrm{es} \&$ tlng $=\mathrm{es}$.

González, S. (2014). La investigación y su aporte al conocimiento: La experiencia de Enfermería en la Facultad de Estudios Superiores Zaragoza de la UNAM. Enfermería universitaria, $17(2)$, 45-46. Recuperado de http://www.scielo.org. 
$\mathrm{mx} / \mathrm{scielo}$.php? script=sci arttext\&pi$d=S 1665-70632014000200001 \& \mid n-$ $\mathrm{g}=\mathrm{es} \& \operatorname{lng}=\mathrm{es}$.

Hernández, R., Fernández, C. y Baptista, P. (2010). Metodología de la investigación (5 ed.). México: McGraw-Hill.

Hernández, I., Mora, J. y Luna, J. (2017). Universidad y empresa: un binomio de responsabilidad social en el siglo XXI. Tendencias. Revista de la Facultad de Ciencias Económicas y Administrativas, 18(1), 145-158.

Huayanay-Espinoza, C. A., Sánchez-Orellana, R., Flores-Córdova, G., Bringas-Delgado, R., Pérez-Romero, F., \& Huicho, L. (2018). La agenda de investigación para la educación superior en el Perú: Hacia el diseño de políticas públicas basadas en evidencia. Revista Digital de Investigación en Docencia Universitaria, 12(1), 301-306. doi: http:// dx.doi.org/10.19083/ridu.12.742

Inquilla, J., Calsina, W. C. y Velazco, B. (2017). La calidad educativa y administrativa vista desde dentro: caso Universidad Nacional del Altiplano - Puno -Perú 2017. Comuni@cción, 8(1),5-15. Recuperado en 03 de junio de 2019, de http://www. scielo.org.pe/scielo.php? script $=$ sci arttext\&pid =S2219-71682017000100001\&ln$\mathrm{g}=\mathrm{es \&} \operatorname{lng}=\mathrm{es}$

Jiménez, V. (2018). La formación de investigadores en la Universidad. ACADEMO, 5(1), 1-2. DOI: 10.30545/academo.2018.enejun. 1

Li-fang, M. F., Tao, D., \& Yunfeng, H. (2019). Emotions and teaching styles among academics: the mediating role of research and teaching efficacy, Educational. Psychology, 39(3), 370-394, DOI: $10.1080 / 01443410.2018 .1520970$

Lizarzaburu, E. (2016). La gestión de la calidad en Perú: un estudio de la norma ISO 9001 , sus beneficios y los principales cambios en la versión 2015. Universidad \& Empresa, 18 (30), 33-54.

Manfra, M. M. (2019). Action Research and Systematic, Intentional Change in Teaching Practice. Review of Research in Education, 43(1), 163-196. https://doi.or$\mathrm{g} / 10.3102 / 0091732 \times 18821132$
Martí, J., Calderón, A. y Fernández, A. (2018). La Responsabilidad Social Universitaria en Iberoamérica: análisis de las legislaciones de Brasil, España y Perú. Universia, 24(9), 107-124.

Masoud, R., \& Hiwa, W. (2018). The impact of research practice on professional teaching practice: Exploring EFL teachers' perception. Cogent Education, 5(1). DOI: 10.1080/2331 186X.2018.1480340

Ministerio de Educación de Colombia (2015). Recuperado de: http://www.mineducacion. gov.co/cvn/1665/w3-article-264648.html

Miranda, S. (2016). La gestión directiva: un concepto construido desde las comprensiones de los directivos docentes de las escuelas públicas bogotanas. RIDE. Revista Iberoamericana para la Investigación y el Desarrollo Educativo, 7(13), 562-589. Recuperado en 03 de octubre de 2018, de http://www. scielo.org. mx/scielo. php? script $=$ sci arttext\&pid = S2007-74672016000200562\&ln$\mathrm{g}=\mathrm{es} \& \mathrm{t} \operatorname{lng}=\mathrm{es}$.

Muñoz, D. y Araya, D. (2017). Los desafíos de la evaluación por competencias en el ámbito educativo. Educação e Pesquisa, 43(4), 1073-1086. DOl: https://dx.doi. org/10.1590/s1678-4634201706164230

Muñoz, M., \& Garay, F. (2015). La investigación como forma de desarrollo profesional docente: Retos y perspectivas. Estudios pedagógicos (Valdivia), $41(2), 389-399$. DOl: https://dx.doi.org/10.4067/S071807052015000200023.

Ortega, R., Veloso, R. y Hansen, O. (2018). Percepción y actitudes hacia la investigación científica. ACADEMO, 5(2), 101-109. DOI: http://dx.doi.org/10.30545/academo.2018.jul-dic.2

Plasencia, M. (2017). Gestión administrativa y calidad de la enseñanza universitaria, en la Facultad de Ciencias Administrativas, de la Universidad Nacional del Callao (Tesis de maestría). Universidad César Vallejo, Lima-Perú.

Piscoya, L. (2015). Retos y perspectivas de la investigación universitaria en el Perú. Alma máter, 2(2), 47-68. 
Proto, F. (2017). Gestión universitaria y transferencia del conocimiento científico. Buenos Aires, Argentina: FAIA.

Rico, A. (2016). La gestión educativa: Hacia la optimización de la formación docente en la educación superior en Colombia. Sophia, 12(1), 55-70. Recuperado en 03 de octubre de 2018, de http://www.scielo.org.co/ $\mathrm{pdf} / \mathrm{sph} / \mathrm{v} 12 \mathrm{nl} / \mathrm{v} 12 \mathrm{nla0}$.pdf

Rivera, C. G., Espinosa, J. M., \& Valdés, Y. D. (2017). La investigación científica en las universidades ecuatorianas.: Prioridad del sistema educativo vigente. Revista Cubana de Educación Superior, 36(2), 113-125. Recuperado en 16 de octubre de 2019, de http:// scielo.sld.cu/scielo.php? script=sci arttext\&pid =S0257-4314201700020001 1\&ln$\mathrm{g}=\mathrm{es} \& \mathrm{t} \operatorname{lng}=\mathrm{es}$.

Rodríguez, E. (2017). Los desafíos estratégicos para la gestión de la investigación universitaria en la sociedad del conocimiento. Ingeniare. Revista chilena de ingeniería, 25(3), 362365. DOI: https://dx.doi.org/10.4067/ S0718-33052017000300362.

Segredo, A. (2016). Aproximación teórica a la evolución, teorías, enfoques y características que han sustentado el desarrollo de las organizaciones. Revista Cubana de Salud Pública, 42(4). Recuperado de http:// scielo.sld.cu/scielo.php? script=sci arttext\&pid =S0864-34662016000400009\&ln$\mathrm{g}=\mathrm{es} \& \mathrm{lng}=\mathrm{es}$.

Soto, R. (2015). La Tesis de maestría y doctorado en 4 Pasos ( $2^{a}$ ed.). Lima, Perú: DIOGRAF.

Superintendencia Nacional de Educación Superior Universitaria. (2020). Licenciamiento. Recuperado de https://www.sunedu.gob. pe/licenciamiento-institucional/

Superintendencia Nacional de Educación Superior Universitaria (2018). Informe bienal sobre la realidad universitaria. Recuperado de https://www.sunedu.gob.pe/informe-bienal-sobre-realidad-universitaria/

Tamayo, L. (2010). Metodología de la investigación científica. Argentina: Limusa.

Tinoco, O. y Vizarreta, O. (2014). Extensión universitaria, proyección social y su relación con la investigación y formación profesional en el marco del proceso de acreditación universitaria en la Fll. Producción y gestión, 17(1), 39-45.

Valderrama. S (2013). Pasos para elaborar proyectos de investigación científica. 5 a. Ed. Lima: San Marcos, 2013. 495 pp. ISBN: 978-612-302-878-7

Veliz, F., Alonso, A., Fleitas, M. y Alfonso, D. (2016). Una gestión universitaria basada en los enfoques de gestión de proyecto y por proceso. Revista Electrónica Educare, 20(3), 1-17. Recuperado de http://www.redalyc. org/pdf/1941/194146862023.pdf

Wyatt, M., \& Dikilitaş, K. (2015). English language teachers becoming more efficacious through research engagement at their Turkish university. Educational Action Research, 24(4), 550-570. DOl: https://doi.org/10.1 080/09650792.2015.1076731.

Yuan, R., \& Lee, I. (2015). Action research facilitated by university-school collaboration. ELT Journal, 69(1), 1-10. DOI: https://doi. org/10.1093/elt/ccu031. 